\title{
A Retrieval Method of CAD Semantic Model Based on Design Intention
}

\author{
Qinyi Ma ${ }^{1, a^{*}}$, Lihua Song ${ }^{1, b}$, Dapeng Xie ${ }^{1, c}$, Maojun Zhou ${ }^{1, d}$ \\ ${ }^{1}$ Department of Mechanical Engineering, Dalian Polytechnic University, Dalian 116034, China \\ aonlypony@163.com, b18340855043@163.com, ‘xiedapeng1105@126.com, ddlmjy@hotmail.com
}

Keywords: 3D model, Semantic retrieval, Design intent, Ontology semantic tree

\begin{abstract}
With the development of 3D modeling technology, the number of 3D models has been increasing and people have been paid more and more attention to the 3D model retrieval technology. This paper presents a CAD semantic model retrieval method based on design intent, which is based on the design intention annotation of 3D model. Firstly, the three-dimensional model ontology semantic tree is built on the basis of modeling information, analyze information and manufacturing information. Then the model semantic trees are built according to the ontology semantic tree and then the similar nodes are returned by comparing the similarity between the target retrieval set and the semantic tree nodes. Finally, the semantic similarity of the model is calculated according to the return nodes and system returns the similar model to complete the search.
\end{abstract}

\section{Introduction}

The 3D models have been widely used in various industries, such as machinery manufacturing, architectural design, molecule biology and so on. Because it is closer to human perception and thinking mode [1], the 3D model data is called the fourth generation multimedia data following the voice, image, and video. The 3D model database has been established, such as the digital Michelangelo project of art database, the general model database PSB (established by Princeton University in America ) [2], the national design libraries composed of the CAD model, mechanical design model and building construction model and so on. Three-dimensional model retrieval technology can help users find the needed 3D model quickly and accurately and reuse the 3D model data, so the 3D model retrieval technology has been paid more and more attention.

Product design mostly evolved from existing products. The difference between so-called initial design, variables design and adaptive design is the degree of utilization of original product. To effectively shorten the product development cycle, designers are always more willing to transform design on proven mature product [3]. The retrieval methods of 3D model are from keyword-based retrieval to content-based retrieval and to the latest semantic-based retrieval method [4]. In the process of content-based retrieval, system only considers the underlying features and the retrieval results can not satisfy human needs because of the semantic gap between the underlying features and the high-level semantics of human beings [5]. The purpose of model retrieval is to reuse the model, so it is very important to capture the design intent accurately of the 3D model. However, the model does not have semantic annotation in the content-based retrieval process and the user needs to understand the semantic information contained in the model by themselves which leads the model reuse efficiency is low. Based on the above reasons, the three-dimensional model retrieval method based on semantic is gradually appeared.

The retrieval method based on the semantic retrieves the model by text, so the semantic annotation information of the 3D model needs to be attached to the 3D model and then the low-level geometric data of the 3D model can be transformed into the high-level knowledge representation. As one of the most direct retrieval methods, its difficulty lies on the semantic expression and use. This paper presents a retrieval method of CAD semantic model based on design intention. Design intent is designer's purpose and intention in the problem-solving process, which are gradation concepts and relationship between the concepts integrated the design objectives, expected functions, the constraints, structural programs, etc. and is a comprehensive reflection of 
design decisions and principles [6]. Given that the products must meet certain functional requirements, design intent is reflected in the entire product life cycle [7]. Firstly, the three-dimensional model ontology semantic tree is built on the basis of modeling information, analyze information and manufacturing information by the mapping method of intent domain-feature domain-information domain [8]. The ontology semantic tree can't cover the all design intentions and it can be extended according to the actual application. The ontology semantic tree is shown in Fig. 1:

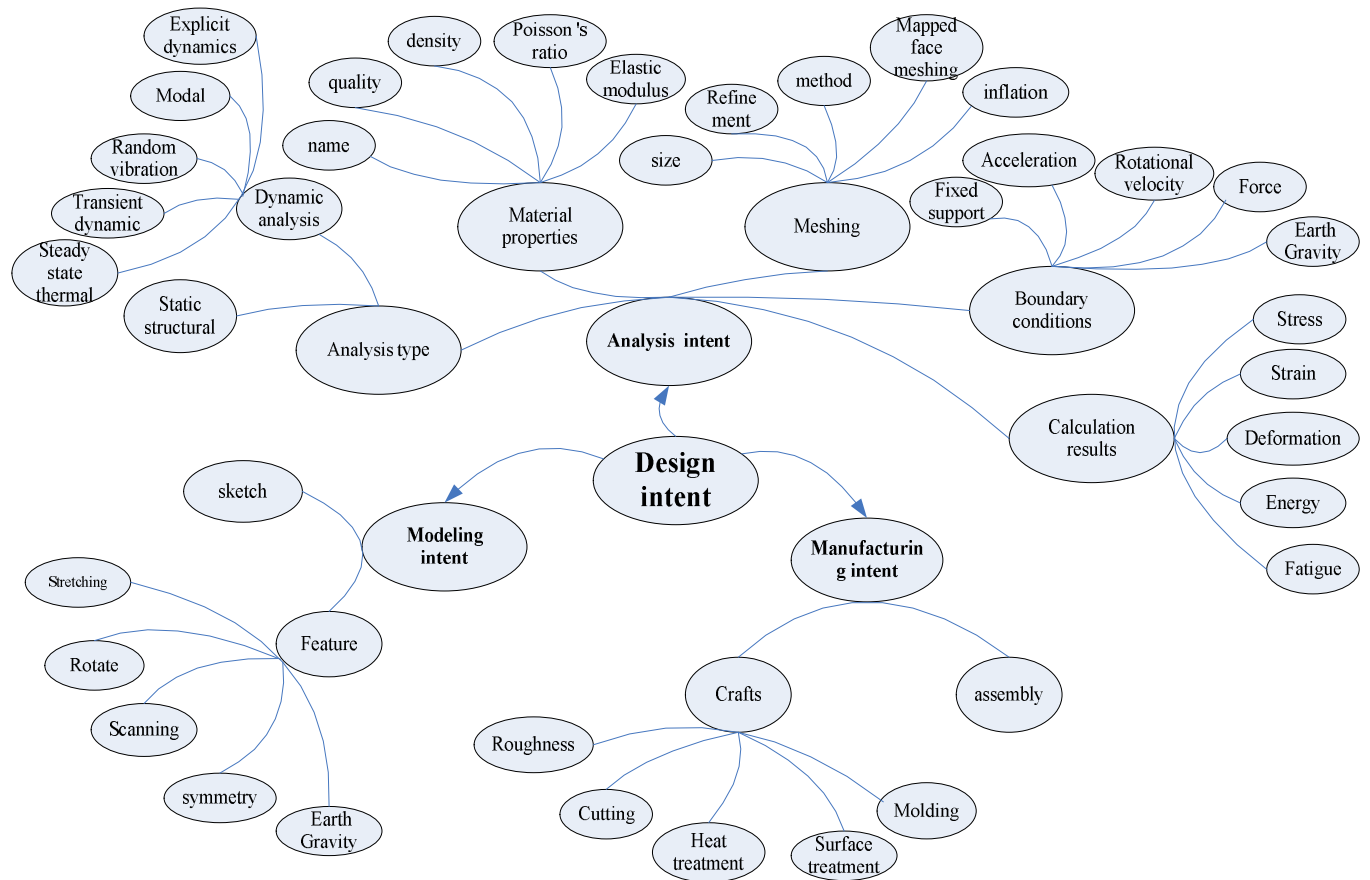

Fig. 1 Ontology semantic tree model.

\section{Semantic Retrieval Process}

The core of semantic-based 3D model retrieval is to describe the 3D model by semantic tree. System searches the 3D model with high similarity by comparing the similarity between the retrieval target words and the semantic tree nodes. Fig. 2 shows a typical semantic-based 3D model retrieval framework:

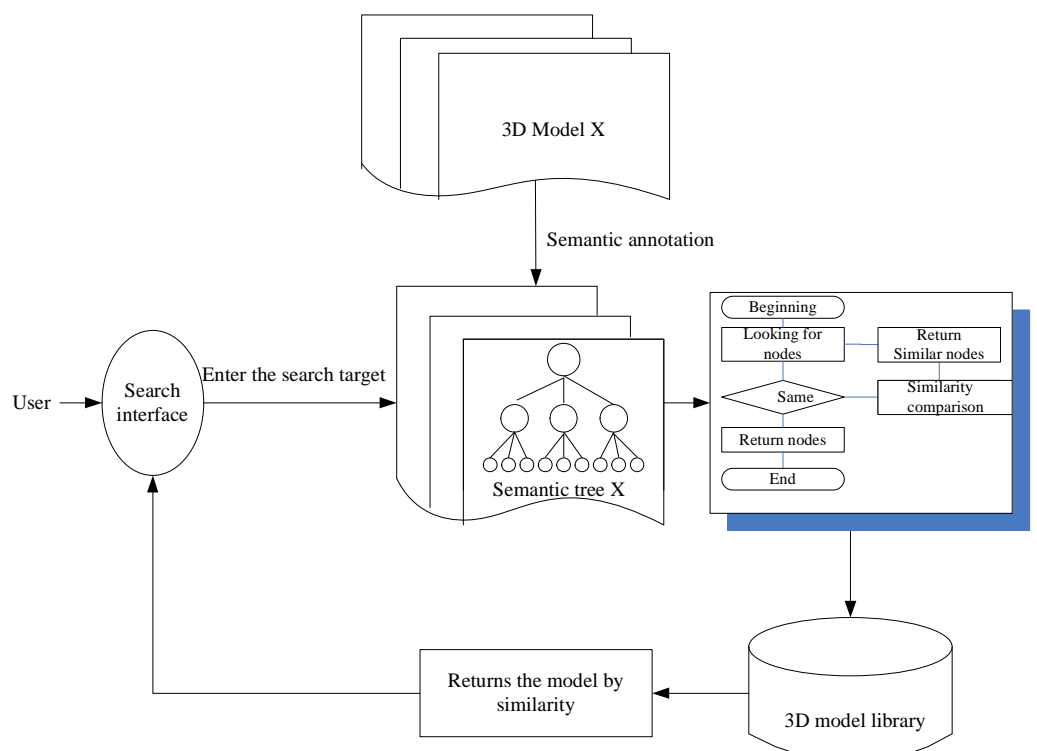

Fig. 2 System retrieval framework. 
(1) Firstly, the user inputs the target search word set $\mathrm{Q}\left(q_{1}, q_{2}, \ldots, q_{k}\right)$, then system compares the semantic similarity between the entered target words and the nodes in the semantic tree $\mathrm{T}\left(\mathrm{t}_{1}, \mathrm{t}_{2}, \ldots, \mathrm{t}_{\mathrm{w}}\right)$ and returns corresponding semantic annotation information $A\left(\mathrm{a}_{1}, \mathrm{a}_{2}, \ldots, \mathrm{a}_{\mathrm{r}}\right)$ of the same or similar nodes and their sub-nodes.

(2) Calculating the similarity of the 3D model according to the returned semantic annotation and returning the similar three-dimensional model $\mathrm{M}\left(\mathrm{m}_{1}, \mathrm{~m}_{2}, \ldots, \mathrm{m}_{\mathrm{n}}\right)$. The results try to reflect the retrieving target more accurately and comprehensively.

System retrieval process shown in Fig. 3:

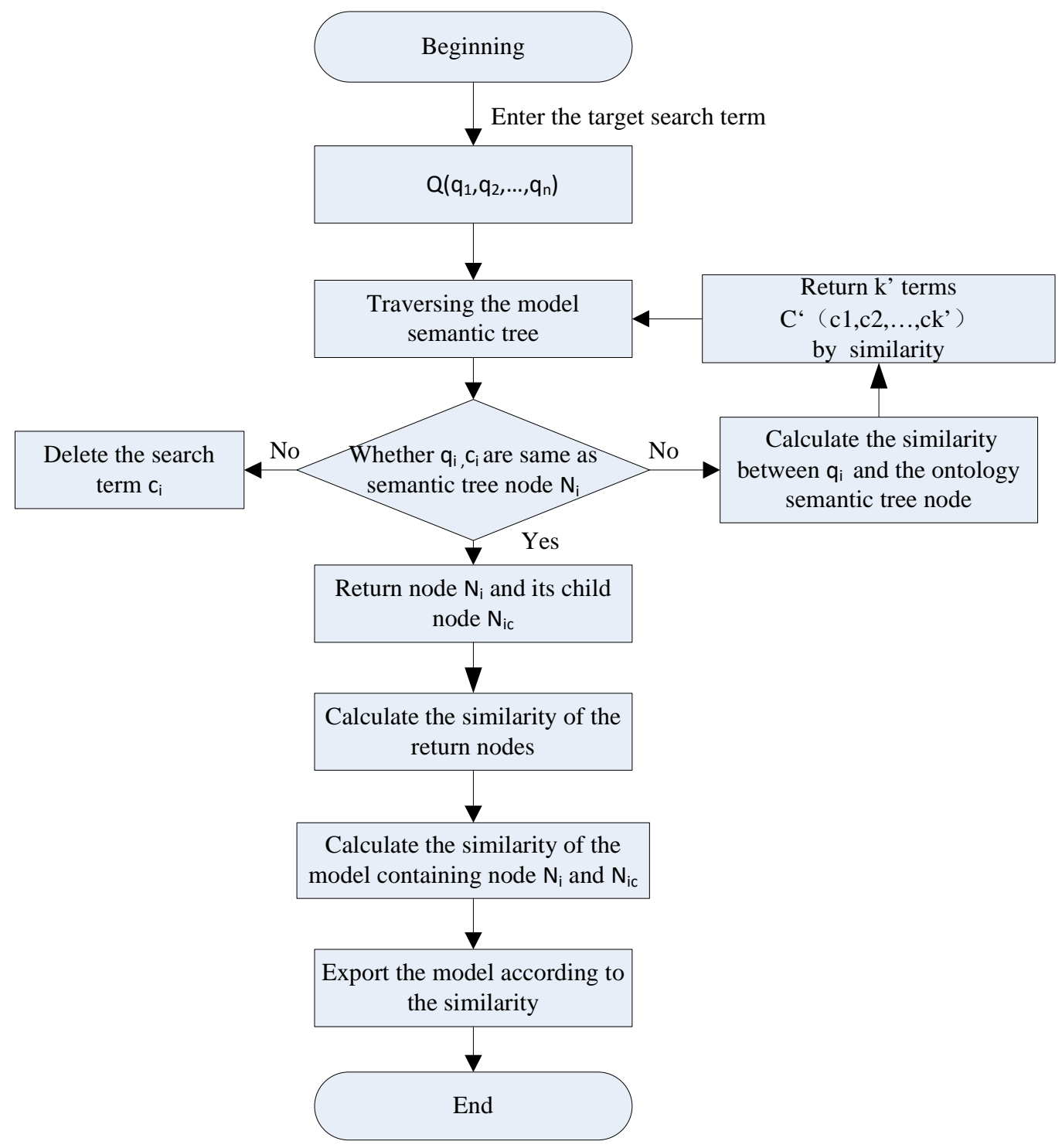

Fig. 3 Retrieval process

To sum up, the 3D model semantic annotation is the basis of the semantic retrieval of the 3D model and the construction of the semantic tree is a prerequisite for $3 \mathrm{D}$ model retrieval. In this paper, semantic tree is constructed on the basis of model annotation semantic classification. The model semantic tree $\mathrm{T}$ is flexible to construct, add and delete tree nodes in real time according to different applications, so the annotation information in each tree does not necessarily contain all the information of the ontology semantics tree. 3D model is annotated part of the information based on its modeling features, analysis features and manufacturing features. The node $\mathrm{N}$ in the semantic tree represents the semantic annotation information of the model and is classified according to the different annotation categories. It is the inclusion relation between the parent node and its child nodes, and the leaf node is an intention unit that can't be subdivided. 


\section{Semantic Similarity Comparison Method}

In order to compare the semantic similarity between the target search term and the model semantic annotation in the model library, both the target search term and the model semantic annotation are based on the description of the 3D modeling information, analysis information and manufacturing information. Firstly, the three-dimensional model retrieval index is built according to the ontology semantic tree to achieve retrieval model and improve retrieval efficiency. The user can input one or more target words $Q\left(q_{1}, q_{2}, \ldots, q_{k}\right)$ by index and then system compares the similarity between the search term $\mathrm{q}$ with the model semantic annotation a and obtains similar semantic annotation words to return them to user.

Semantic similarity comparison method is as follows:

(1) Traversing the semantic tree of the model and matching each element in the target retrieval set with the tree node, and when it happens that the target retrieval word is same as the node of the model semantic tree $\mathrm{T}$, the similarity degree of the node and the target search term is recorded as 1 and then the corresponding semantics of the node $N_{i}$ and its child node $N_{i c}$ is returned. Supposed there are $\mathrm{m}$ child nodes, the corresponding similarity $S_{c}$ of each child node is $1 / \mathrm{m}$. Supposed there are $\mathrm{p}$ returned annotations.

(2) If the target search word $q_{i}$ is not same as any node in the semantic model trees, system will find a node which is same or similar semantic as $q_{i}$ based on the ontology semantic tree and the node and its child nodes (n) are returned, recording as $\mathrm{C}^{\prime}\left(\mathrm{c}_{1}, \mathrm{c}_{2}, \ldots, \mathrm{c}_{\mathrm{k}^{\prime}}\right)$. The $\mathrm{C}^{\prime}$ will be used as the new target search term and matched with the tree nodes using the method 1). The corresponding annotation information to the node and its child node are returned. Supposed there are $\mathrm{p}^{\prime}$ returned annotations.

This paper adopts the method of the hierarchical relationship among words to calculate the similarity between the search term $\mathrm{q}_{\mathrm{i}}$ and the ontology semantic tree node. The semantic similarity is calculated based on the relationship between the upper and lower positions of words. When compare relationship between the retrieval target words and the model semantic annotations, this paper adopts the method similar with the Jiang \& Conrath method [8]. For the search term $\mathrm{q}_{i}$, system calculates the information content ic value based on the number of the nodes in the ontology semantic tree, the formula is as follows:

$$
i c_{w n}(c)=\log \left(\frac{\operatorname{hypo}(c)+1}{\max _{w n}}\right) / \log \left(\frac{1}{\max _{w n}}\right)=1-\frac{\log (\operatorname{hypo}(c)+1)}{\log \left(\max _{w n}\right)}
$$

The function hypo returns the number of hyponyms for a semantic concept. $\max _{w n}$ is a constant which is set to the maximum size of the concept in this class. The denominator is equal to the concept containing the maximum amount of information, and the final result ic value is in the $[0,1]$.

The semantic similarity between words $c_{i} / c_{j}$ is calculated as follows:

$$
\operatorname{sim}_{j c n}\left(c_{i}, c_{j}\right)=1-\left[\frac{i c_{w n}\left(c_{i}\right)+i c_{w n}\left(c_{j}\right)-2 \times \operatorname{sim} m_{r e s}\left(c_{i}, c_{j}\right)}{2}\right]
$$

Among it, $\operatorname{sim}_{r e s}\left(c_{i}, c_{j}\right)=\max _{c \in S\left(c_{i}, c_{j}\right)} i c_{r e s}(c), c_{i} \in W, c_{i} \in S$ 。

Then the semantic similarity of each word in the set $C^{\prime}$ is:

$$
S_{C}, \quad=\operatorname{sim}_{j c n} \times \mathrm{S}_{c} \text {. }
$$

\section{D Model Return Method Based on Semantic Similarity}

A good return model strategy can make the semantic information contained in the return model cover all the semantics of the target search term, and will return the first W model to users. Every returned semantic information (There are $p+p^{\prime}$ returned annotations) in retrieval system have a corresponding similarity value (each model corresponding $\mathrm{p}, \mathrm{p}^{\prime}$ value is different).The $3 \mathrm{D}$ model retrieval system returns $3 \mathrm{D}$ model set $\mathrm{M}\left(\mathrm{m}_{1}, \mathrm{~m}_{2}, \ldots, \mathrm{m}_{\mathrm{n}}\right)$ and the model similarity value is the 
semantic similar sum of corresponding $\mathrm{p}+\mathrm{p}^{\prime}$ returned annotation information:

$$
\mathrm{S}_{\text {model }}=\sum_{\mathrm{t}=1}^{\mathrm{p}} \mathrm{S}_{\mathrm{C}_{\mathrm{t}}}+\sum_{\mathrm{u}=1}^{\mathrm{p}^{\prime}} \mathrm{S}_{\mathrm{C}_{\mathrm{u}}}
$$

The sum is bigger, the relationship between the model and the retrieval target is bigger, else the relationship is smaller between the model and the retrieval target set.

\section{Conclusion}

This paper presents a CAD semantic model retrieval method based on design intent. Firstly, the three-dimensional model ontology semantic tree is built on the basis of modeling information, analyze information and manufacturing information by the mapping method of intent domain-feature domain-information domain. Then model semantic tree and semantic tree database are constructed according to the ontology semantic tree. Finally, the same or similar nodes will be returned by comparing the similarity between the target retrieval terms and the semantic tree nodes and the related models are output to user by the 3D model return method. This method can improve the retrieval efficiency of 3D model and reflect users' needs more comprehensively and accurately.

\section{Acknowledgements}

This work was financially supported by National Natural Science Foundation of China (No.51305051), the program for Liaoning Excellent Talents in University (No.LJQ2015007), the Liaoning Province Natural Science Foundation (No.201602060), and the Liaoning Province Natural Science Foundation (No.2014026006).

\section{Literature References}

[1] Y. B. YANG, H. LIN, Q. ZHU. Content-Based 3D Model Retrieval: A Survey, Chinese J. Comput. 27 (2004) 1297-1310.

[2] F. Thomas, et al. A Search Engine for 3D Models. ACM Trans. Graph. 22 (2003) 85-105.

[3] J. F. WANG, M. S. LU. MBD based product assembly dataset and its applications, Manuf. Automat. 35 (2013) 78-82.

[4] Y. Zhang, The Implementation and Researches of 3D Model Retrieval System Based on Semantics. Changchun: Jilin University, 2009.

[5] J. H. Tang, Z. J. Zha, D. C. Tao, et al. Semantic-Gap-Oriented Active Learning for Multilabel Image Annotation. Image Proc. IEEE, 21(4) (2012) 2354.

[6] Z. S. Wang, T. Ling, W. R. Duan. Knowledge push technology based on design intent modeling. Comput. Integrat. Manuf. Syst. 21(3) (2015) 606-617.

[7] W. Xu. Study on Conceptual Modeling Technology Based on Design Intent Capture. Beijing, Tsinghua University, 2010.

[8] S. F. Jiang, S. Chen. Integration of capturing design intent and parametric design. Comput. Integrat. Manuf. Syst. 4 (2011) 726-731. 